Proceedings of SPIE, Vol. 4394, April 2001

\title{
Environmental Factors that Impact the Performance of a Seismic Land Mine Detection System
}

\author{
Gregg D. Larson ${ }^{a}$, James S. Martin ${ }^{\mathrm{a}}$, Waymond R. Scott, Jr. ${ }^{\mathrm{b}}$ and George S. McCall II ${ }^{\mathrm{c}}$ \\ ${ }^{a}$ School of Mechanical Engineering \\ ${ }^{\mathrm{b}}$ School of Electrical and Computer Engineering \\ ${ }^{\mathrm{c}}$ Georgia Tech Research Institute \\ Georgia Institute of Technology \\ Atlanta, GA 30332
}

\begin{abstract}
A system has been developed that uses high frequency seismic waves and non-contacting displacement sensors for the detection of land mines. The system consists of a moving displacement sensor and a stationary elasticwave source. The source generates elastic waves in the earth. These waves propagate across the minefield where they interact with buried mines. The sensor measures the displacements at the earth's surface due to the passage of the waves and the interactions of the waves with mines. Because the mechanical properties of the mine are different from those of the earth, the surface displacements caused by the interaction are distinct from those associated with the free-field propagation of the waves. This provides the necessary cue for mine detection. The system has been demonstrated in a controlled laboratory environment, and efforts are currently underway to transition this work into field tests. Moving the experimental effort into the outdoor environment is a critical milestone toward the ultimate goal of this research effort, which is the design of a field-operable mine detection and classification system. There are many issues associated with this transition. Foremost among these is the propagation characteristics of seismic waves in the field environment and, particularly, the mechanisms that limit the energy which can be coupled into the seismic signal that is used to search for mines. To investigate this, a field site was selected which reasonably emulates the existing laboratory experimental model, and a series of measurements was undertaken to determine the effects of environmental factors at both sites on the generation and propagation of seismic waves. At both sites, strong non-linearity was observed which limited the energy content of the incident signal.
\end{abstract}

Keywords: land mine, mine detection, non-linearity, seismic waves

\section{INTRODUCTION}

Seismic/elastic techniques show considerable promise for the reliable detection of all types of buried mines, even low-metal anti-personnel mines. The reason for this is that mines have mechanical properties that are significantly different from soils and typical forms of clutter. For example, the shear wave velocity is approximately 20 times higher in the explosive and the plastics used in typical mines than in the surrounding soil [1]. In addition, mines are complex mechanical structures with a flexible case, a trigger assembly, air pockets, etc. The complex structure gives rise to structural resonances, non-linear interactions, and other phenomenology that is atypical for both naturally occurring and man-made forms of clutter. Thus, this phenomenology can be used to distinguish a mine from clutter.

A system has been developed at Georgia Tech that uses a radar-based displacement sensor for the local measurement of seismic displacements without physically contacting the soil surface [2-3]. The non-contact nature of this sensor makes the system capable of interrogating the soil surface near or immediately above a mine. This substantially increases the measurable effects of the mine's presence over schemes which rely on elastic waves scattered by the mine to propagate to a remote sensor location [4]. Figure 1 depicts the present system configuration. The sensor shown in the figure is bistatic. Both bistatic and monostatic configurations have been 
studied. The system consists of the non-contact sensor and the ground coupled seismic source. The source (an electrodynamic shaker resting on a narrow foot) excites an incident signal composed primarily of a Rayleigh surface wave with measurable energy also propagating in bulk shear and compressional waves. The incident signal travels across the minefield and excites motion of soil near the surface that includes the buried mines. The sensor measures this motion over an array of measurement locations. Sensor data are processed in both space and time domains to yield an image of the minefield [5]. To interrogate the earth over the range of typical mine sizes and burial depths, the incident signal has a spectrum from $50 \mathrm{~Hz}$ to $1,000 \mathrm{~Hz}$.

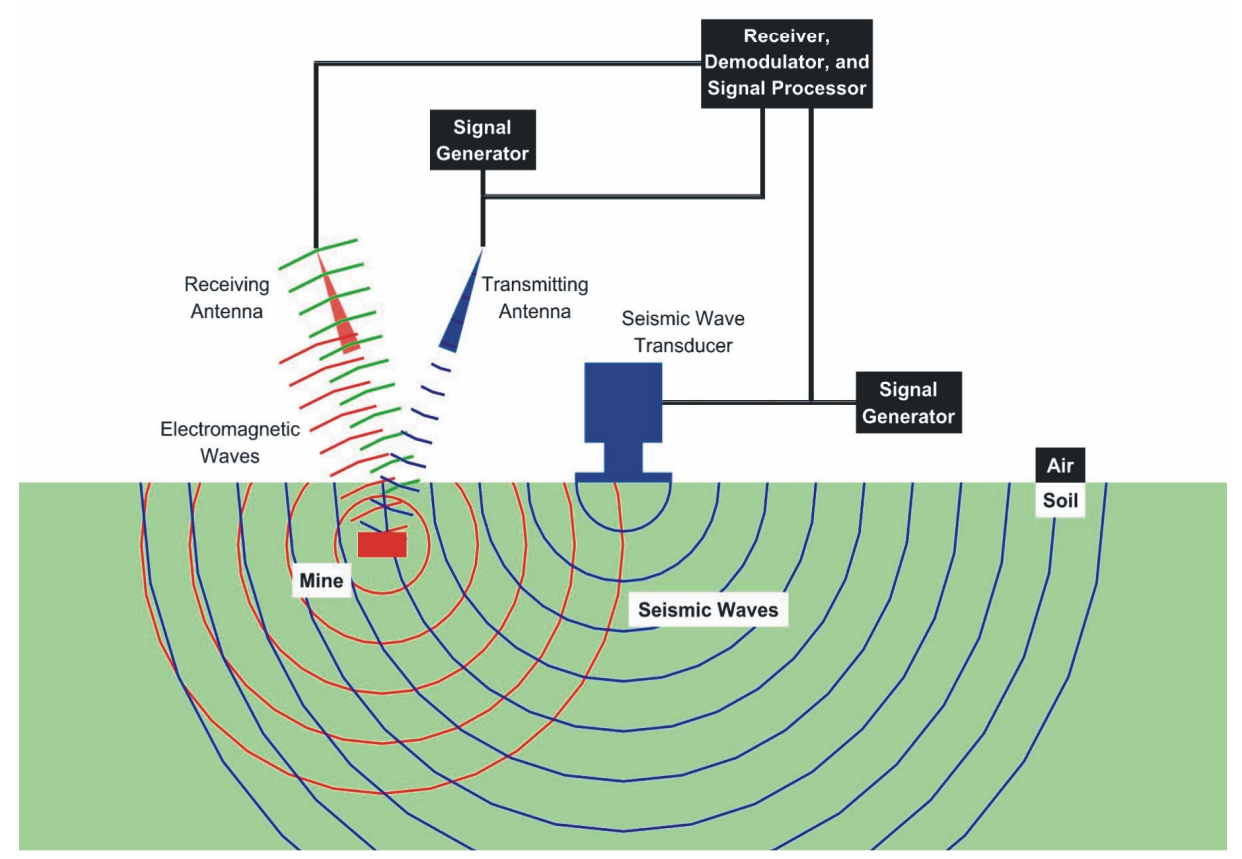

Figure 1: Schematic of a Seismic Mine Detection System

The seismic mine detection system has been investigated experimentally over the past 3 years using a series of laboratory models of increasing size and complexity. Damp compacted sand was selected as the medium for these models because of the ease and repeatability it offered for the burial of mines, mine simulants, and clutter objects. Anecdotal evidence exists in the literature to suggest that this is a reasonable model for soils [6,7]. The published data are sparse on the range of frequencies and depths that are of interest for this problem and are limited to the characterization of soils within the linear regime. Scaling of published experimental data and theoretical results involving very different frequency ranges and material parameters requires a sufficient experimental database to justify the comparison. Additionally, results generated with the laboratory model suggest phenomena with no obvious precedent in the literature. These non-linear effects can be observed at relatively low drive levels (displacements on the order of $1 \mu \mathrm{m}$ ). They include dispersion of surface guided waves, an inverse relationship between amplitude and group velocity, and amplitude-dependent loss. Whether these are typical of the natural environment or peculiarities of the laboratory model is yet to be determined. The work presented here represents the first attempt to address some of these questions.

The radar-based displacement sensor is not presently configured for field tests. Since the topic of current interest is environmental characteristics rather than sensor development, it was not deemed necessary that the system sensor be used for the field measurements. The baseline sensors that were used for the laboratory-to-field comparisons were, therefore, ground coupled accelerometers. These have the advantage of having comparable sensitivity to the radar sensor over most of the band of interest while being waterproof and robust in a comparatively hostile environment. The accelerometers also offer a simple method for the construction of a 
physical array, whereas the radar sensor is currently a single sensor that is scanned to form a synthetic array. The synthetic array cannot easily distinguish between long-timescale changes, such as tidal motions of the water table, and short-timescale changes, such as dispersion of a propagating waveform. Unlike the laboratory environment, these factors cannot be controlled in the field. Vibra-Metrics VMI-9002A accelerometers encapsulated in syntactic foam with $100 \mathrm{mV} / \mathrm{g}$ sensitivity were used in conjunction with custom-built preamplifiers and power supply decouplers for their internal charge amplifiers. Data were acquired without low-pass filters on 24 channels sampled at $8 \mathrm{KHz}$ with 12-bit resolution in all of the accelerometer measurements. Anti-aliasing filters were not used in the data acquisition system because previous experiments in the linear regime had demonstrated that the need for these was obviated by the rapid attenuation of high frequencies with propagation distance in the sand. Although the Nyquist frequency was 2 octaves above the highest transmit frequency $(1 \mathrm{KHz})$ and well above the dominant spectral components of the background noise, aliasing problems introduced by the lack of filters complicated the analysis of the data. Aside from this problem, the accelerometer system offered a signal to noise ratio (SNR) equivalent to that achieved by the radar sensor in the laboratory at $100 \mathrm{~Hz}$. Since the radar sensor responded to displacement rather than acceleration, there was a $12 \mathrm{~dB}$ per octave SNR improvement associated with the accelerometers at high frequencies and a corresponding degradation at low frequencies. An additional relative improvement in the accelerometers' high frequency SNR is offered by its small integration area on the ground's surface $\left(3 \mathrm{~cm}^{2}\right)$ compared with approximately $9 \mathrm{~cm}^{2}$ illuminated by the radar sensor (depending on the sensor's height).

\section{LABORATORY AND FIELD MEASUREMENTS IN THE LINEAR REGIME}

Figure 2 shows the laboratory model and the field site. The laboratory model, which has been described in detail in a previous paper [2], is a $1.5 \mathrm{~m}$ deep wedge-shaped tank filled with 50 tons of masonry sand that is kept damp and compacted with a water table maintained $50 \mathrm{~cm}$ below the surface. The field test site is at the Navy beach adjoining the campus of the Naval Postgraduate School (NPS) in Monterey, California. This has been the site of ongoing micro-seismic experiments undertaken at NPS and is quite near a beach location extensively characterized by Bachrach and Nur in 1995 [8]. Several different accelerometer array configurations were used at both sites. The cross configuration depicted in Figure 2 represents the largest of these. Tides varied the water height at the field site both during and between experiments. The dimensions shown are typical, although depth changes of up to $50 \mathrm{~cm}$ occurred during the experiments. This translated into horizontal changes of up to $10 \mathrm{~m}$. Some of the experiments were performed at low tide on the harder sand below the high water mark. Others were performed at high tide on the softer dry sand above the high water mark. Similar propagation speeds were observed in both cases, although the wet sand results more closely matched those of the laboratory model. Figure 3 depicts waterfall plots generated by pulse compression of data taken at both the laboratory and field sites. The data shown here were not measured directly but were reconstructed from measured transfer functions. For each of these data sets, the raw data, which were generated using a 4 second linearly-swept-frequency chirp covering the entire band of interest, were used to synthesize the response of the system to a temporal discrete waveform with a spectrum centered in the middle of the band of interest. In this case, the compressed drive signal was the first derivative of a Gaussian pulse centered at $400 \mathrm{~Hz}$. The graphs in Figure 3 indicate the similarity between the laboratory model and field site. Two distinct arrivals are observed at each site. The first of these (any apparent earlier arrivals are caused by noise-induced processing artifacts) arrives with a group velocity of $170 \mathrm{~m} / \mathrm{s}$ to 183 $\mathrm{m} / \mathrm{s}$, which is consistent with observed P-wave velocities [8] and with observations of leaky surface waves [9] at similar sites. The series of small arrivals immediately following the first arrival may account for the latter observation, although both wave types may not exist with measurable amplitude in the data. The second and largest pulse arrival propagates with a speed of $87 \mathrm{~m} / \mathrm{s}$ to $90 \mathrm{~m} / \mathrm{s}$ and is due to the Rayleigh surface wave. The peaks of this waveform have been clipped in the figure in order to display both wave types in a single graph. This speed is consistent with the other reported observations of Rayleigh waves in both sand and hard soil [7,9]. The coda associated with the Rayleigh wave arrival is longer and more complex in the field data than in the laboratory

because of the variety of surface and subsurface inhomogeneities at the field site. These include footprints, buried flotsam, and a water table that was inclined with respect to the ground surface and non-uniform due to wave 
action. Although identical pulse shapes were used with both data sets, there are also some differences in the shapes of the received signals: the data from the laboratory model have lower spectral content than the field data. This is due to a difference in the frequency response of the two different transducers that were used in these tests. A MB Electronics 20-lb. shaker was used as a source for the field tests, but this was damaged in shipping and replaced by a 100-lb. VTS shaker for the later laboratory measurements. Although the Rayleigh wave pulse has been clipped in these plots, dispersion is apparent in both the laboratory and field data. In a homogenous, isotropic, semi-infinite medium, these waves would be non-dispersive. The most likely cause for this dispersion is a vertical inhomogeneity in the sand. This may be due to the depth dependence of water content (as hypothesized in [8]) or to the lithostatic force on the lower strata (as in the earth's crust on large length scales [10]).
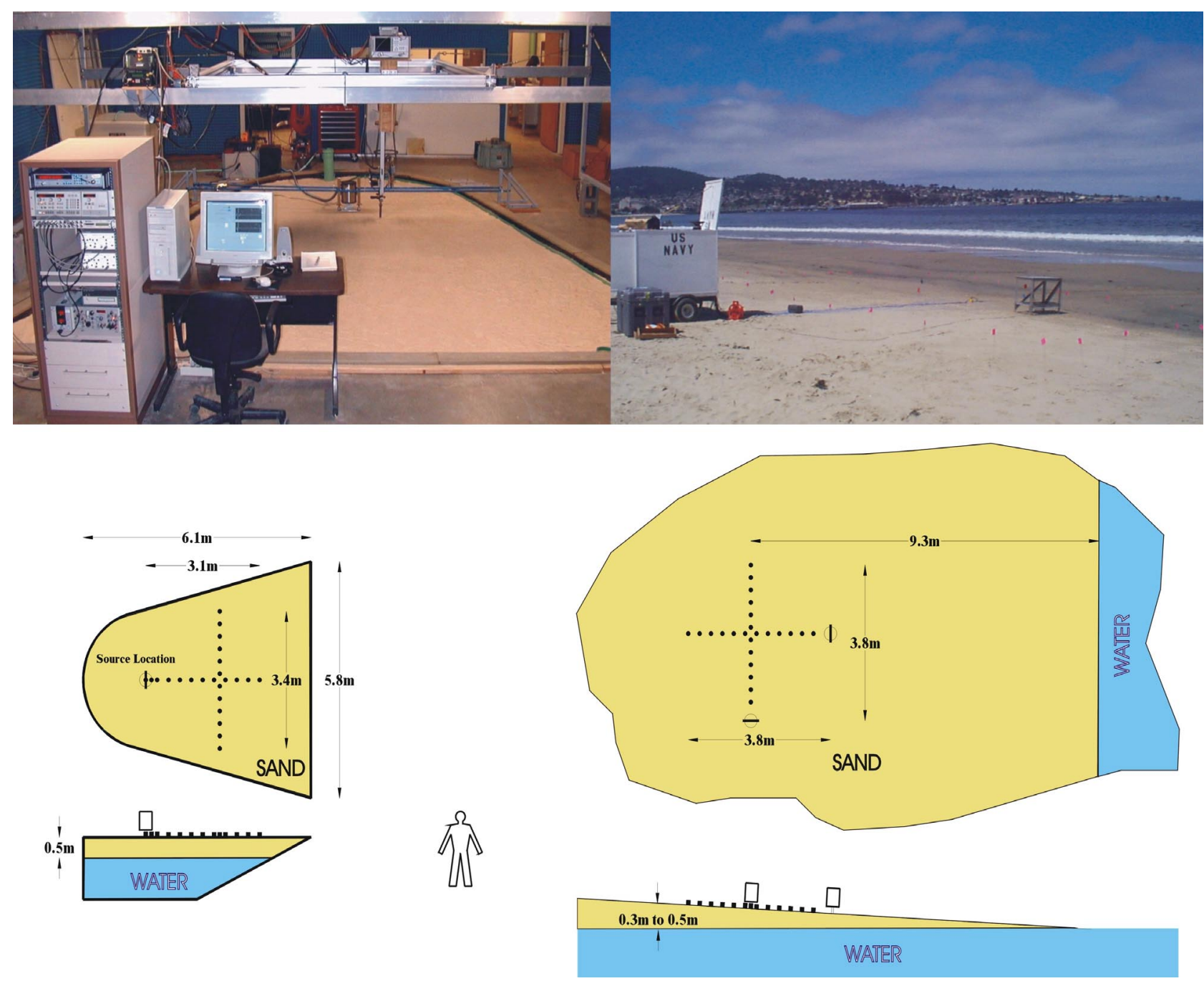

Figure 2: Experimental Models, (A) the Laboratory Model and (B) the Field Test Site 

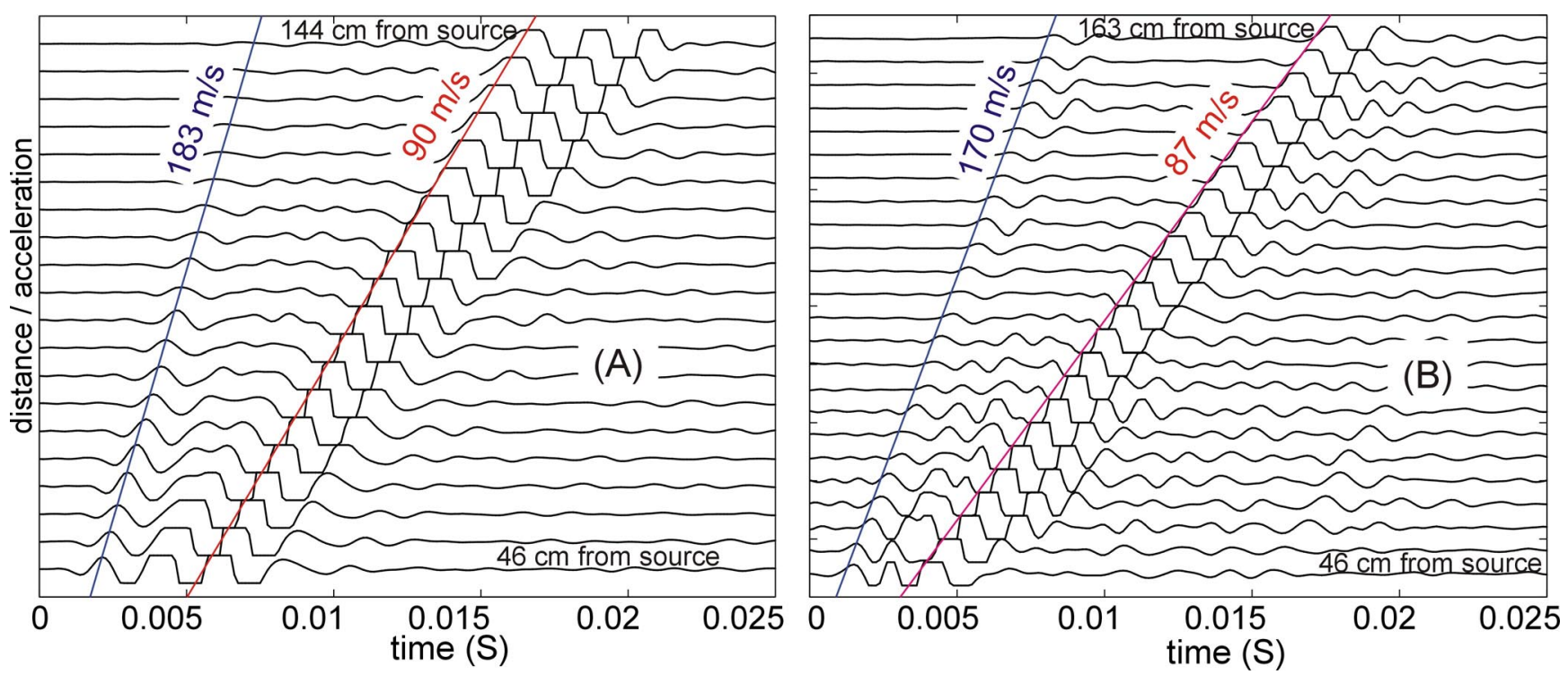

Figure 3: Propagation of a Seismic Pulse, in the Laboratory Model (A) and at the Field Test Site (B)

A second difference between the field site and the laboratory model is the horizontal anisotropy in the wave propagation introduced by the incline of the water table. Figure 4 shows a comparison of measurements made perpendicular to the direction of wave propagation for two cases from the field site: first, for a wave propagated along the shoreline, and second, for a wave propagated directly inland. The waveforms represent only the surface wave pulse (the faster arrivals are time gated out) and are not clipped, but they have been normalized by their peak values to correct for the directivity of the source. The inland propagation measurement shows a very strong top/bottom symmetry along the array as one would expect for an isotropic medium. The along-shore measurement, however, shows a distinctly different pulse shape arriving at the shoreward end of the array. This dispersion may be due to the narrowing dimension of wave guide between the surface and the water table or to a lowering of the wave speed with water content below complete saturation. This was observed by Bachrach and Nur [8] for P-waves in a similar environment.
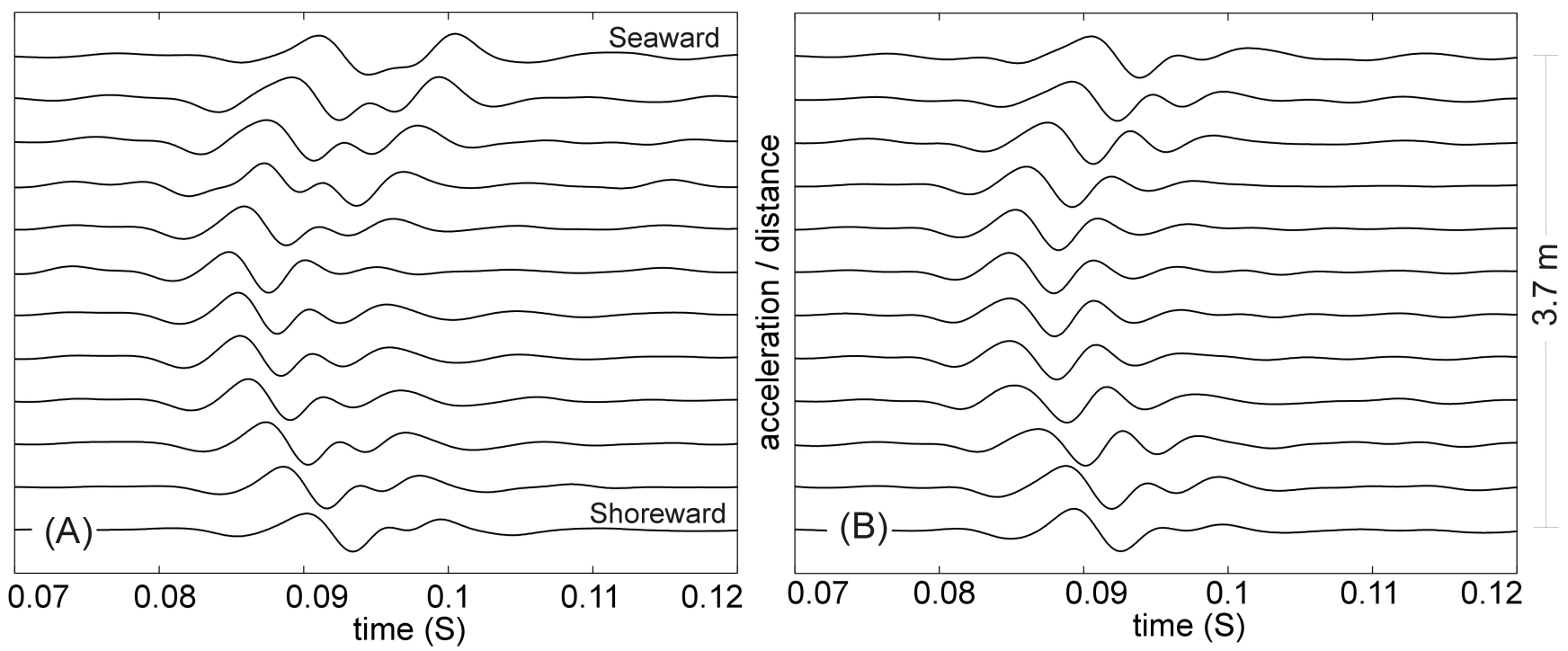

Figure 4: Comparison of Wave-Front Transects: Propagation Parallel to the Beach (A) and Shoreward

Propagation (B) 
It is also interesting to note that a third difference between the laboratory model and the field site was in the observed ambient noise fields. This difference, surprisingly, favored the field site. In spite of wave action, wind, and foot traffic, the noise floors measured at the field site were entirely due to the instrumentation noise of the measurement system below $500 \mathrm{~Hz}$. In contrast, the noise floor in the laboratory model has several distinctive features that can be attributed to artificial sources and couple into the model through the foundation of the building that houses it. On average, the laboratory measurements were made over a noise floor which was 4 to 6 $\mathrm{dB}$ higher than the field site's noise floor in the $50 \mathrm{~Hz}$ to $1000 \mathrm{~Hz}$ band.

\section{HARMONIC GENERATION AND NON-LINEAR STEEPENING}

As mentioned previously, the incident signals used for these experiments were linearly-swept-frequency chirps. This makes it possible to separate non-linear effects in the long-time domain using a short-time Fourier transform as a function of long times (i.e. a spectrogram). Figure 5 depicts such a spectrogram for the signal received at an accelerometer located $50 \mathrm{~cm}$ from the seismic source on dry sand (above the high water mark) at the field test site. The lowest of the positively sloped ridges on the contour plot represents the fundamental component of the drive signal. The ridges with steeper slopes represent each of the higher harmonics generated in the source to sensor path as indicated by the numbers on the plot. Thus, it is a relatively simple matter to distinguish the $200 \mathrm{~Hz}, 300 \mathrm{~Hz}$, and $400 \mathrm{~Hz}$ components generated by non-linear mechanisms from the same spectral components contained in the original drive signal because these effects are not concurrent on a long timescale. It is clear from the figure that the system is highly non-linear with more than 8 harmonics distinguishable above the noise floor. The aliasing problem mentioned earlier is also clear in this figure. Although the drive signal contains no energy above $1 \mathrm{KHz}$, the harmonics of the drive signal higher than the second contain energy above the Nyquist frequency of $4 \mathrm{KHz}$. Thus, the ridges appear to reflect from the upper frequency bound of the figure. At the times when these aliased signals are coincident with the drive or other harmonics, the two effects cannot be separated in post-processing. Both plots in Figure 5 depict the same signal spectrogram. In Figure 5B, the dynamic range has been compressed and converted to a gray scale, which uses only the bottom 20 $\mathrm{dB}$ of the $60-\mathrm{dB}$ dynamic range available above the noise floor. From this figure, it is apparent that sub-harmonics of the drive signal are also generated at very low levels in the wave propagation. This may be a feature unique to the dry sand or may not have been manifest above the noise floor in the data acquired below the high water line, which showed a similar content of overtones.
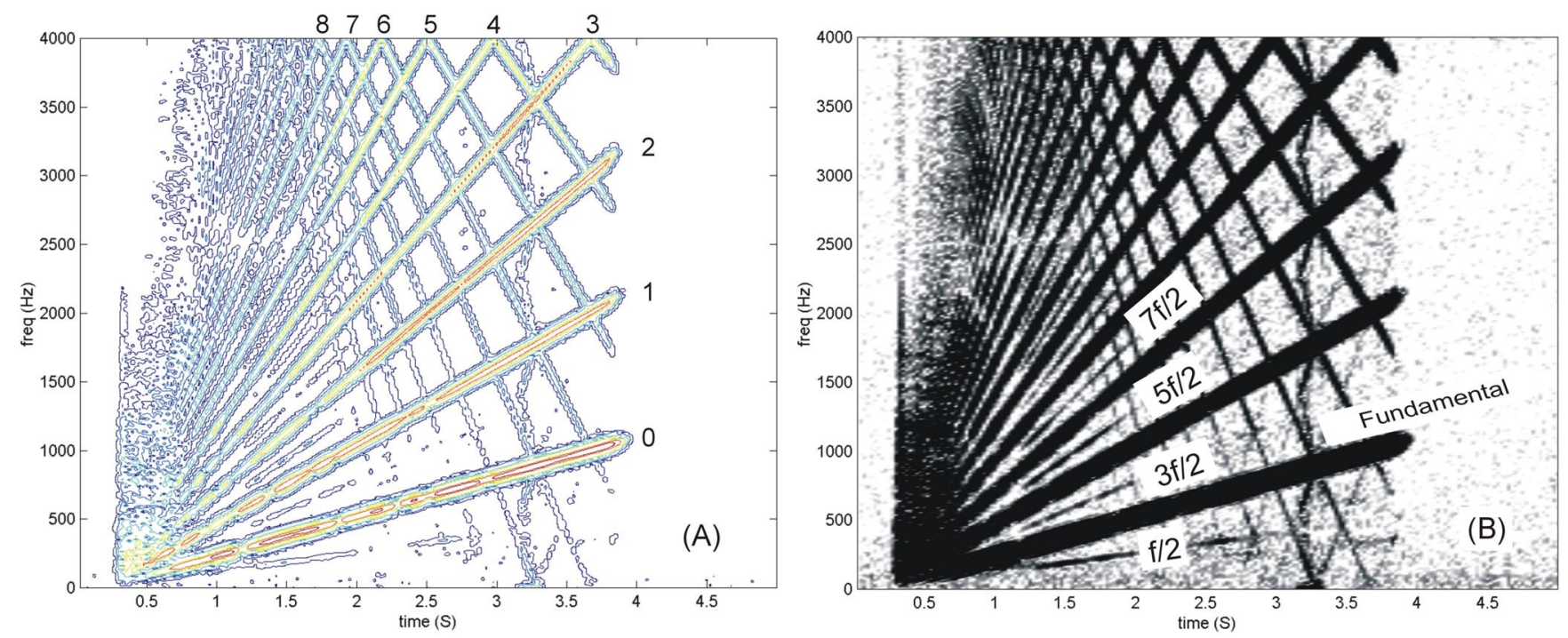

Figure 5: Spectrogram of Acceleration Signal Measured $50 \mathrm{~cm}$ from the Seismic Source at the Field Site on Dry Sand, Depicted on a $60 \mathrm{~dB}$ Scale (A) and on a $20 \mathrm{~dB}$ Scale at the Bottom of the Dynamic Range Measured (B). 
It is difficult to attribute the generation of the higher harmonics and sub harmonics solely to the nonlinearity of the sand. Source non-linearity and non-linearity in the source-to-sand coupling may also play a role in the observed phenomena. Spectral analysis of multiple points along the propagation path shows that, across most of the measurement region ranging from $0.35 \mathrm{~m}$ to $5.0 \mathrm{~m}$ from the source, the propagation appears to be linear in the data acquired at the field site. The question remains, however, as to whether or not there is a distinguishable exchange of energy between fundamental frequencies and overtones within the measured region. This is somewhat difficult to address because geometrical spreading reduces the signal with distance, and the higher frequencies are attenuated very quickly in the sand. Harmonics, therefore, cannot be seen to grow along the propagation path in the field test data unless normalized by the fundamental and then only over the nearest 3 to 4 measurements. However, an examination of a similar experimental setup from the laboratory reveals evidence to support propagation path non-linearity as the cause of the harmonics observed in the field tests. Figure 6 shows a comparison between spectra that were observed in the sand and at the source. Although harmonics of the drive signal exist at the source, they have clearly been amplified relative to the fundamental by the first $35 \mathrm{~cm}$ of propagation through the sand. Only three overtones are observable in the source motion and six can be seen in the sand. Further evidence in support of this is available from an examination of waveforms from the laboratory model in the time domain. Figure 7 depicts measurements made using a $396 \mathrm{~Hz} \mathrm{CW}$ drive signal. A single cycle of motion has been plotted at the source and at distances of $30.5 \mathrm{~cm}$ and $244 \mathrm{~cm}$ away from the source in the sand. All the signals have been shifted in time and have been scaled to a common peak amplitude in order to emphasize changes in the waveform shape over delay and attenuation. Although very little distortion is observable in the source motion, the signal has converted to a series of sharp high frequency impulses of downward motion separated by broad low frequency positive phases. This transition occurs over the first $30 \mathrm{~cm}$ of propagation. Losses, then, strip the high frequency energy from the signal, and it has nearly returned to the shape of the drive at the $244 \mathrm{~cm}$ range, which is effectively in the "old age" region for the evolution of this waveform. The distortion observed at $30 \mathrm{~cm}$ is not consistent with the formation of shock fronts predicted by the Fubini solution for a onedimensional compressional wave [11]. It is, however, consistent with the numerical predictions of Zabolotskaya for the vertical displacement component of a non-linear Rayleigh wave in a homogeneous solid (steel)[12].
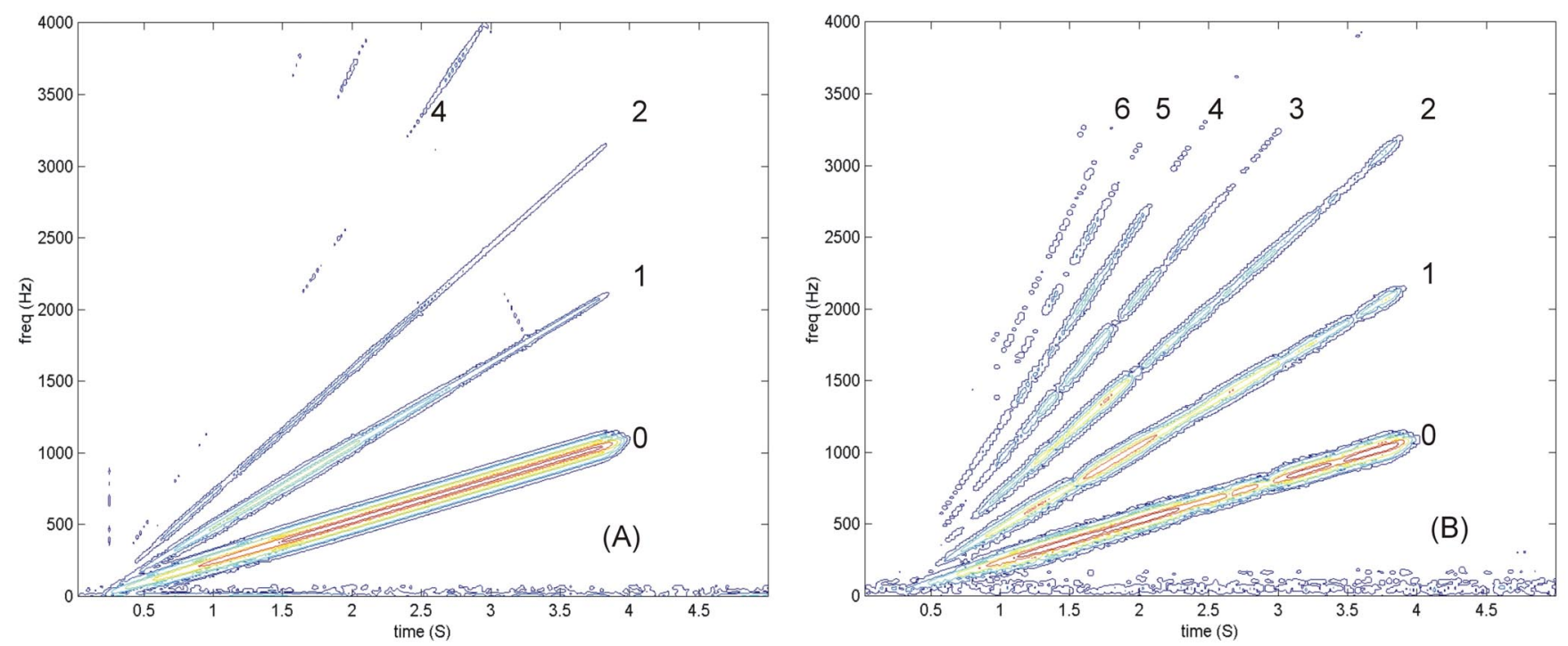

Figure 6: Spectrograms of Measured Acceleration on a $60 \mathrm{~dB}$ Dynamic Range Measured at the Source (A) and in the Sand $30.5 \mathrm{~cm}$ from the Source (B) in the Laboratory Model Experimental Model 


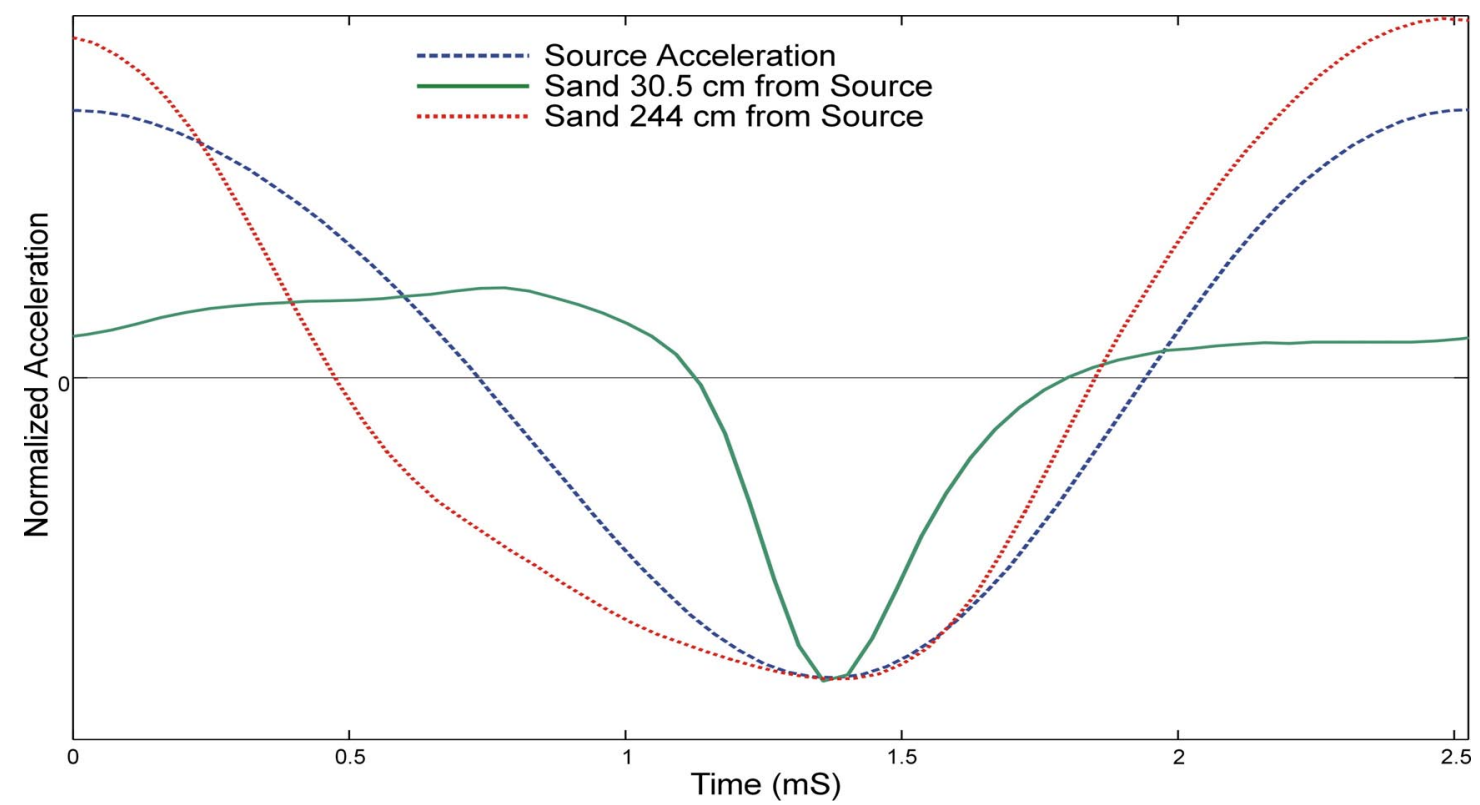

Figure 7: Steepening of $396 \mathrm{~Hz}$ CW Waveform with Propagation Distance

\section{SATURATION AND SPALL}

There are two obvious limits to the transfer of energy into seismic waves: saturation and spall. Under saturation, marginal level additions to the drive signal are entirely converted into higher harmonics and attenuated so that they make no contribution to the signal level received far from the source where the fundamental component dominates. Thus, an increase in drive signal does not produce a corresponding increase in the amplitude of the propagating elastic wave. Detachment of the source from the sand surface, known as spall, is the equivalent of cavitation at the face of an acoustic transducer immersed in a fluid. This occurs when either the biasing force on the shaker contact (the weight of the shaker itself for the data presented here) or the cohesion within the sand below that contact point is inadequate to maintain contact between the source and the sand.

Experimental evidence indicates that each of these mechanisms may limit energy transfer under different circumstances both in the laboratory model and at the field test site. Also, there is some evidence to suggest that the two effects may not be entirely separable in that spalling on a granular scale may be involved in the saturation limit. Spall at the source occurred more readily at the field test site in the tests performed on the dry sand above the high water mark. It was audible during the tests as a rumble midway through some of the frequency sweeps. Figure 8 depicts a spectrogram containing a spall event measured $51 \mathrm{~cm}$ from the shaker source on dry sand; the signals from closer accelerometers clipped in the analog electronics during this event. The event is seen midway through the signal and is easily distinguishable from the harmonic generation, which precedes and succeeds it, because of its broadband nature. Both before and after the event, energy is confined to discrete bands around the drive frequency and its overtones, whereas the rupture of the sand distributes energy evenly over the entire bandwidth of the measurement system and probably beyond this as well. Although the force applied to the contact surface was constant through the drive signal, the contact has obviously been restored after the spall event. This is probably because the sand surrounding the contact was fluidized by the spall event, and the settling of the shaker into the surface improved the contact sufficiently to end the event.

All of the field measurements were performed with transient signals at empirically maximized drive levels; unfortunately, the point at which individual spectral components of the drive signal went into saturation could not be determined from this limited data set. The experiment was therefore repeated in the laboratory model using a pure tone drive at $396 \mathrm{~Hz}$. This is near the middle of the band of interest. The results of this experiment are plotted in Figure 9. Here the measured levels of the fundamental component and the first two harmonics have been plotted for the acceleration measured in the sand at 4 different ranges against that measured at the source. All 
of the curves depict a nominally linear amplitude ratio for source accelerations up to about $1 \mathrm{~g}$. Beyond this, the received signals begin to saturate. Interestingly, the signals measured at $61 \mathrm{~cm}$ and $122 \mathrm{~cm}$ from the source enter a phase where incremental additions to source level result in an actual decrease in measured acceleration of the sand. This may be due to an interference, which occurs when portions of the source contact have fluidized while others have not. It is also possible that the combined effects of dispersion and regeneration of the fundamental frequency by mixing of overtones produces a destructive interference.
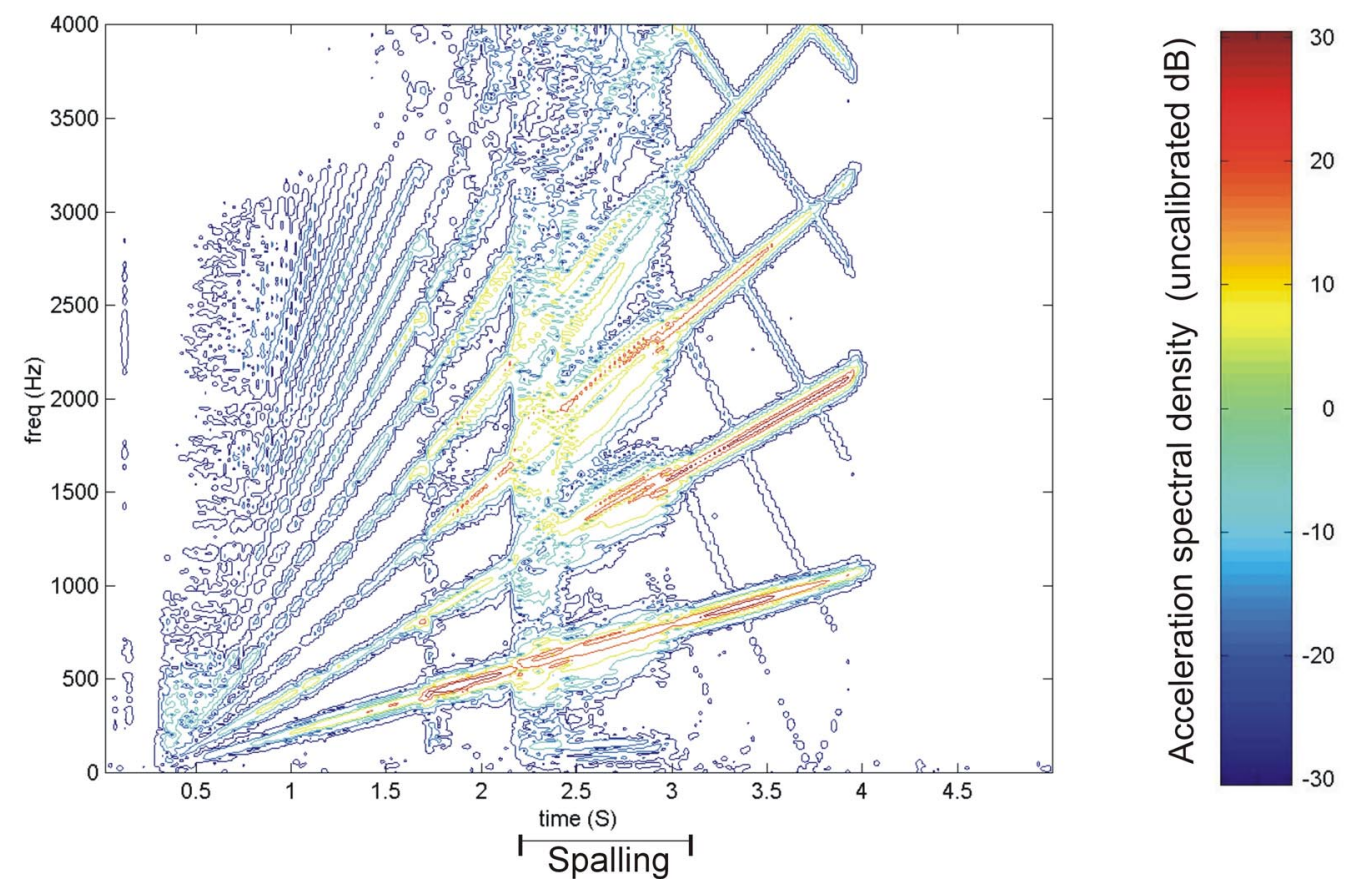

Figure 8: Spectrogram of a Spall Event Measured at the Field Site $46 \mathrm{~cm}$ from the Source on Dry Sand
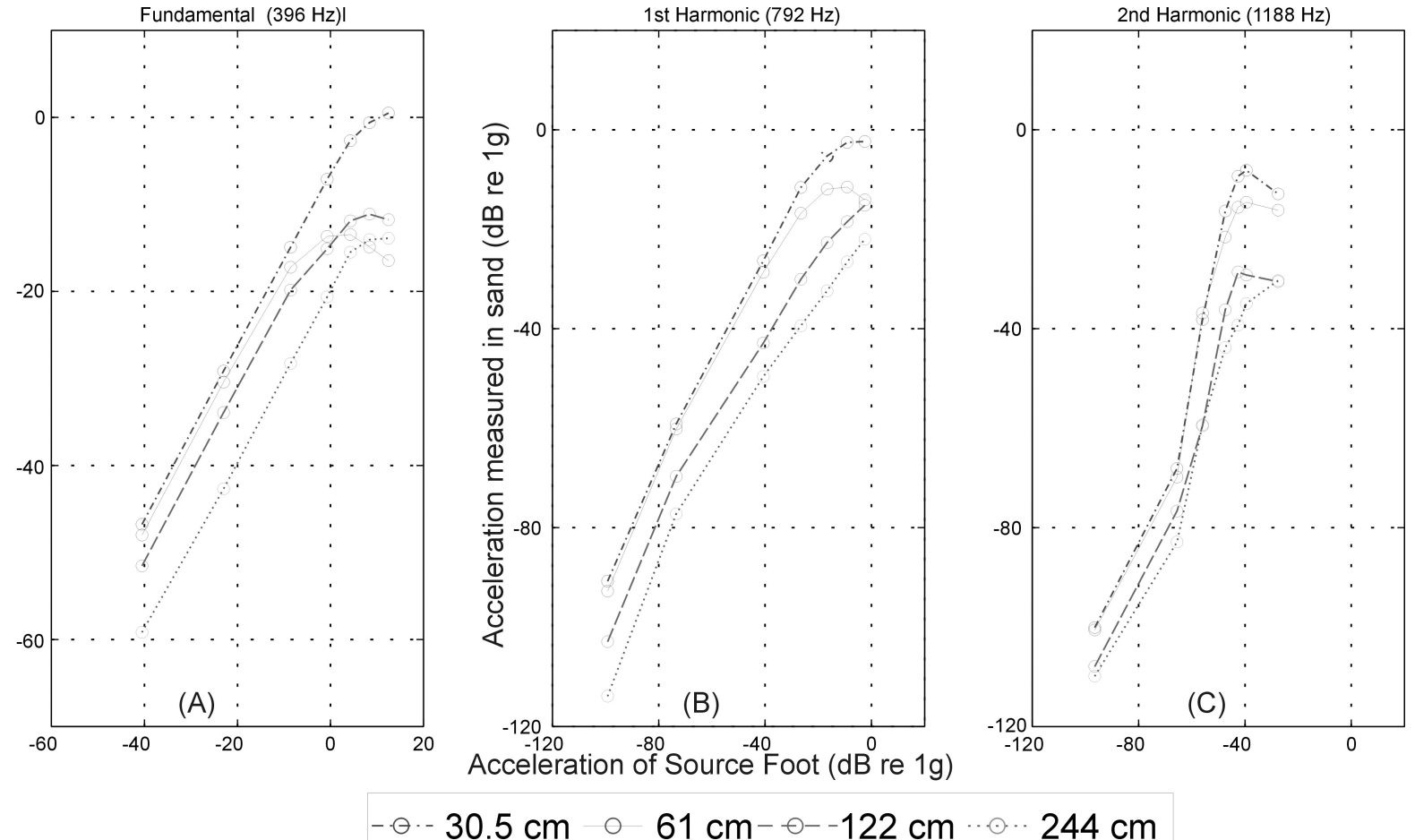

Figure 9: Measured Levels of $396 \mathrm{~Hz}$ Fundamental (A) and Harmonics (B\&C) in Sand vs. Source 


\section{NON-LINEAR DISPERSION}

Dispersion can be observed in measured surface waveforms in most of the data from both the laboratory model and the field site. It is apparent in the change in pulse shape as a function of location shown in Figures 3 and 4. The nature of this dispersion has significant implications for the post-processing of experimental data. Linear dispersion of Rayleigh waves, which can occur as a consequence of the vertical gradients in material properties, is fully accounted for in the pulse compression routine that generated the waveforms depicted in Figures 3 and 4. Non-linear dispersion, however, is not accounted for, since sum and difference frequencies occur at times dependent on pulse shape and cannot be accounted for with a simple impulse response. The differences between these two types of dispersion are illustrated by the laboratory results shown in Figure 10. Here, the acceleration was measured at four separate locations using a differentiated Gaussian pulse with a center frequency of $450 \mathrm{~Hz}$ as the original drive signal rather than reconstructing the pulse response in post-processing. What is depicted are raw data, although 1000 pulses have been averaged together to build up the signal-to-noise ratios for each measurement. The horizontal separation in Figure 10 indicates the four different measurement locations in the model at $30.5 \mathrm{~cm}, 61 \mathrm{~cm}, 122 \mathrm{~cm}$, and $244 \mathrm{~cm}$ along a line away from the source. The magnitude of the offset is arbitrary. Propagation delays of $3 \mathrm{~ms}, 6 \mathrm{~ms}, 16 \mathrm{~ms}$, and $31 \mathrm{~ms}$ have been subtracted before the waveforms were stacked so that all the data could be displayed in a single graph. Each vertical separation between traces indicates an increase of $3 \mathrm{~dB}$ in drive signal amplitude. The signals have all been normalized by their peak acceleration amplitude in order to emphasize changes in pulse shape over acceleration amplitude differences which are approximately three orders of magnitude for the data depicted.

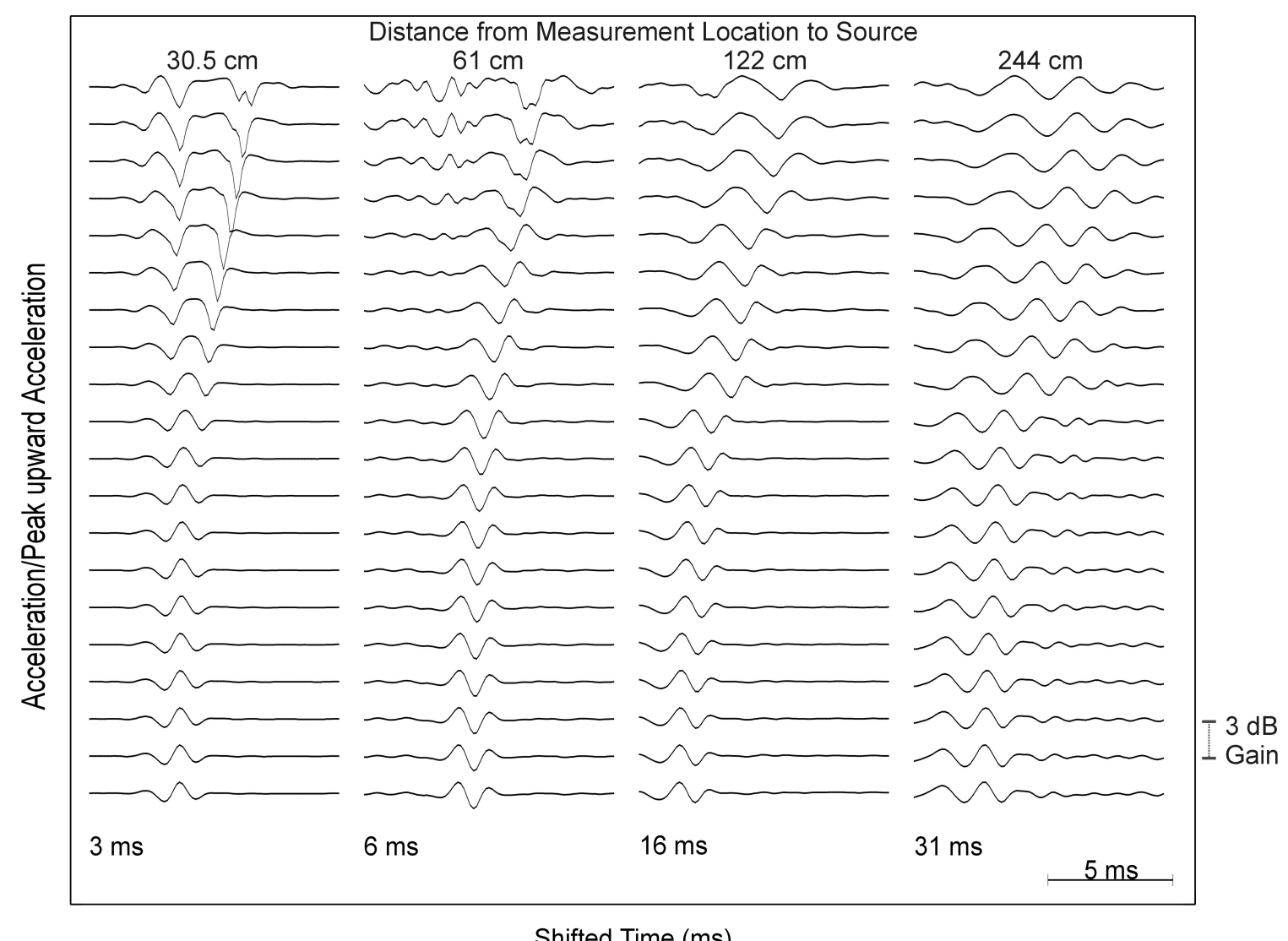

Shifted Time (ms)

Figure 10: Changes in Shape of Rayleigh Wave Pulses with Increasing Drive Level and Propagation Distance

The apparent inversion of the signal between the first and second measurement points at very low drive levels is an indication of linear dispersion. Viewed as a function of position, the peaks and troughs of the signal 
can be seen to propagate through the pulse envelope as this envelope propagates across the sand surface. The obvious change in pulse shape and the delays associated with the pulse arrival at high drive levels are the effect of non-linear dispersion. The troughs of the original pulse can be seen to form two downward acceleration impulses $30 \mathrm{~cm}$ from the source, similar to those depicted in Figure 7 for a continuous drive. These impulses separate in time as drive level is increased, much like the broadening of shock waves observed in air [11]. At greater distances there is, however, a preference toward not only the low frequency components of the original pulse, but also the energy that arrives late in time. This is particularly apparent in the data taken at $61 \mathrm{~cm}$ where the arrival of the pulse is visibly delayed with increasing drive. This inverse relationship between the group velocity and the drive level appears to have no precedent in the literature and will be a focus of further research in this area. The physical processes that mediate this phenomenon are not well understood. Its nature is further obfuscated by the fact that it was originally observed in completely linear pulse compression of an FM sweep in the non-linear regime and was believed to be an artifact of this clearly invalid processing scheme. This implies that the effect is mediated by a dependence of phase velocity on both drive amplitude and frequency, but not by the generation of harmonics, which are time gated out of the pulse compression. It may involve small spatial regions (slip planes or un-compacted zones) where stress concentrations cause local fluidization and lower the average shear strength along the propagation path in a way that recovery cannot occur on the timescale of the excitation. It is also possible that energy preferentially leaks out of the leading portion surface wave into the bulk of the medium. Since all bulk waves propagate with higher velocities than the surface wave, this form of energy loss might preferentially attack the leading edge of the waveform and create the impression of the pulse's slowing down.

\section{CONCLUSIONS}

Measurements have been conducted at a field test site and in a laboratory model that qualify the role played by system non-linearity in limiting the potential range and signal level that can be exploited by a seismic mine detection system. Non-linearity in both the source coupling and the propagation path have been observed and demonstrated to be possible signal limitations. Observed steepening and non-linear dispersion pose potential problems for the post-processing of data that may also limit useable signal levels. Work is currently underway to understand and quantify all of these effects and their potential impact on system operations.

\section{ACKNOWLEDGEMENTS}

This work is supported in part by the Office of Naval Research under contact number N00014-99-1-0995.

\section{REFERENCES}

1. Patel, D.L., "Handbook of Land Mines and Military Explosives for Countermine Exploitation," US Army Belvoir RD\&E Center Technical Report No. 2495, March 1992.

2. Scott W.R., Jr., Larson, G.D. and Martin J.S., "Simultaneous Use of Elastic and Electromagnetic Waves for the Detection of Buried Land Mines," Proceedings of the SPIE: 2000 Annual International Symposium on Aerospace/Defense Sensing, Simulation, and Controls, Orlando, FL, Vol. 4038, April 2000.

3. Scott W.R., Jr., Martin J.S., and Larson G.D., “ Experimental Model for a Seismic Landmine Detection System," IEEE Trans on Geoscience and Remote Sensing. To appear July 2001

4. "Feasibility of Acoustic Landmine Detection: Final Technical Report," BBN Technical Report No. 7677, May 1992. 
5. Behboodian, A., Scott, W.R., Jr. and McClellan, J.H., "Signal Processing of Elastic Surface Waves for Localizing Buried Land Mines," Proceedings of the 33rd Assilomar Conference on Signals, Systems, and Computers, Assilomar, CA, October 1999.

6. Sabatier, J.M., Bass E.H., Bolen, L.N., and Attenborough, K., "Acoustically Induced Seismic Waves," Journal of the Acoustical Society of America, Vol. 80, no. 2, pp. 646-9, August 1986.

7. Westebbe, M., Bohme, J.F., Krummel, H., and Matthews, M.B., "Model fitting and testing in near surface seismics using maximum likelihood in frequency domain," Conference Record of thirty-second Asilomar Conference on Signals, Systems and Computers, Vol. 2, pp.1311-15, November 1998.

8. Bachrach, R, Nur, A., and Dvorkin, J., "High-Resolution Shallow-Seismic Experiments in Sand," "Part I" and "Part II," Geophysics, Vol. 63, No. 4, pg. 1225-40, July 1998.

9. Smith, P., Wilson P.S., Bacon F.W., Manning, J.F., Behrens, J.A., and Muir T.G, "Measurement and Localization of Interface Wave Reflections from a Buried Target," Journal of the Acoustical Society of America, Vol. 103, no. 5, pg. 2333-43, May 1998.

10. Lay T. and Wallace, T.C., "Modern Global Seismology." NY: Academic Press, 1995, pp. 252-63.

11. Beyer, R.T., "Nonlinear Acoustics." NY: Acoustical Society of America Press, 1997, pp. 91-109 and pp. 189202.

12. Zabolotskaya, E.A., "Nonlinear Propagation of Plane and Circular Rayleigh Waves in Isotropic Solids," Journal of the Acoustical Society of America, Vol. 91, no. 5, pp. 2569-75, May 1992. 